\title{
On Modelling of Water Distillation in a Reverse Osmosis Process
}

\author{
Osniman Paulina Maure, Sudi Mungkasi \\ \{osnimanpaulinamaure@gmail.com ${ }^{1}$, sudi@usd.ac.id ${ }^{2}$ \} \\ Department of Mathematics Education,Universitas Sanata Dharma, Yogyakarta, Indonesia ${ }^{1}$, \\ Department of Mathematics, Universitas Sanata Dharma, Yogyakarta, Indonesia ${ }^{2}$
}

\begin{abstract}
In this paper, we solve the water purification equation in a reverse osmosis system of salt water. We shall predict the concentration of salt solution at a given position and time in the process of reverse osmosis. We use the two-dimensional advectiondiffusion equation as the governing equation, which is a partial differential equation. The partial differential equation is converted into ordinary differential equations with a transformation method. Furthermore, in searching for solutions, an analytical approach is used. This approach is successful in computing the concentration of salt in the reverse osmosis process.
\end{abstract}

Keywords: advection-diffusion equation, reverse osmosis, water distillation.

\section{Introduction}

One alternative in water purification systems is to use a reverse osmosis (RO) process. This is because desalination using $\mathrm{RO}$ membranes requires low energy compared to other desalination processes [1]. Reverse osmosis is the process of separating and removing dissolved solids, organic, pyrogenic, submicron colloids, colors, nitrates and bacteria from water using a semipermeable spiral membrane [2]. Membrane is a thin layer that can separate various materials physically or chemically by applying a driving force [3]. In the reverse osmosis process high pressure is applied to the concentrated side (contaminated side) of the membrane. When pressure is applied to this side, pure water will flow through a semipermeable membrane towards the other side of the lower concentration [4]. In reverse osmosis systems, a semipermeable membrane is arranged in layers in one roll as in Figure 1.

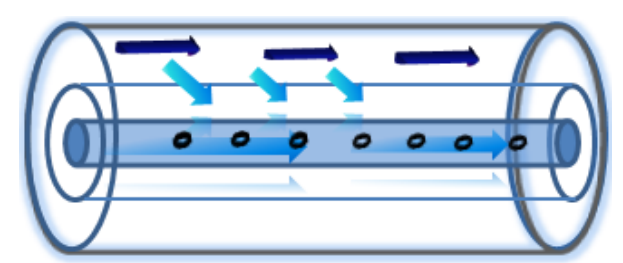

Fig. 1. Semipermeable membrane layers in reverse osmosis

In this process, the solute is deposited in the pressure-filled layer so that pure solvents can flow to the next layer while the water containing the concentration of salt solution will accumulate on the other side of the semipermeable membrane [5]. The process of distributing 
concentration with space and time is described by parabolic type partial differential equations known as advection-diffusion equations [6]. Therefore, we will develop the $2 \mathrm{D}$ advectiondiffusion model by considering the form of a semipermeable membrane in a reverse osmosis system. One-dimensional model cannot capture transverse transport so that a two-dimensional model is needed [7]. This 2D advection-diffusion equation is a partial differential equation. This partial differential equation is the governing equation of many physical phenomena such as fluid dynamics, acoustics, electrostatics, electrodynamics, fluid flow, and elasticity [8]. This equation will be converted into ordinary differential equations with transformation methods. Furthermore, in finding solutions we use an analytical approach. Analytical solutions of an equation are very important in understanding and describing the physical phenomena that occur [9]. This case study topic was previously carried out by Fulford and Broadbridge using analytical analysis [10].

In this paper, the author will explain mathematical modeling and mathematical computation to predict the concentration of salt solution in reverse osmosis process in water purification. This paper is a description in line with Fulford and Broadbridge [10].

The rest of this paper is organized as follows. Section 2-4 consists of mathematical modelling, results, and conclusion.

\section{Mathematical Modelling}

The focus of this problem is on the concentration of salt which is very close to a semipermeable membrane because this is where salt buildup is formed. This is shown in Figure 2 by considering semi-infinite areas $x>0, y>0$ and a semipermeable membrane on $y=0$. The flow of water flows through the channel with speed $v(y)$, parallel to the $x$ axis and the pressure on the membrane accelerates the water out at a known flow rate $q$.

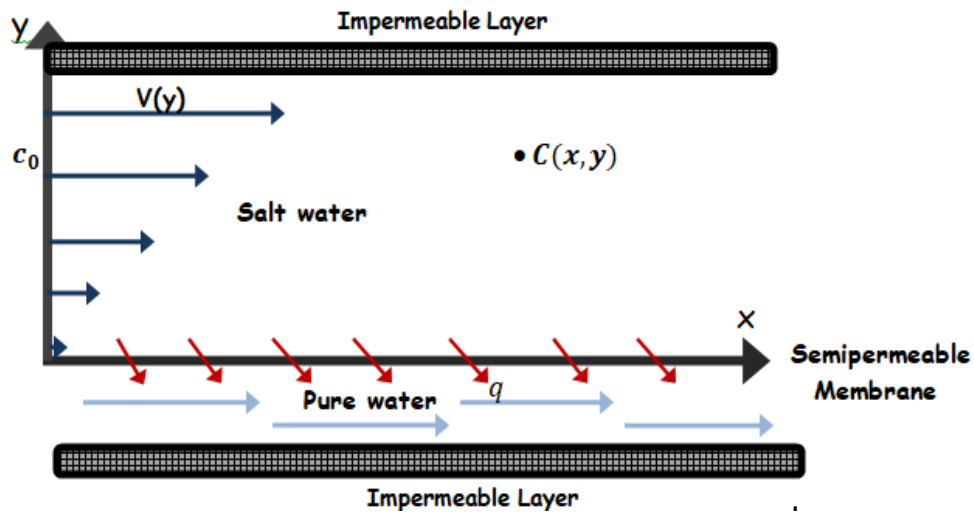

Fig. 2. Advection and diffusion near semi-permeable membranes

The mathematical model for defining salt concentration $C(x, y, z)$ is expressed by the following $2 \mathrm{D}$ advection-diffusion equation:

$$
\frac{\partial C}{\partial t}+v_{1}(y) \frac{\partial C}{\partial x}=D\left(\frac{\partial^{2} C}{\partial x^{2}}+\frac{\partial^{2} C}{\partial y^{2}}\right),
$$


where $v_{1}(y)$ is a horizontal component of velocity that depends on the distance from the membrane whose value is zero on the membrane surface. The salt concentration changes rapidly in the $y$ direction, so as to allow the spread of salt in the $y$ direction, and thus dispersion in the $x$ direction is ignored. Setting $\frac{\partial C}{\partial t}=0$, the governing equation (1) becomes

$$
v_{1}(y) \frac{\partial C}{\partial x}=\frac{\partial^{2} C}{\partial y^{2}} .
$$

The speed of the salt mixture in the membrane is zero. Above the membrane, the speed increases to some speed $v_{0}$. Thus, the velocity close to the semipermeable membrane is

$$
v(y)=\frac{v_{0} y}{h},
$$

where $h$ is the distance from the semipermeable boundary to the center of the channel. Note that the horizontal velocity $v(y)$ is zero at the semipermeabel membrane. Differential equations governing the salt concentration are obtained by substituting equation (3) into equation (2), so that we obtain

$$
y \frac{\partial C}{\partial x}=\alpha \frac{\partial^{2} C}{\partial y^{2}}
$$

where $\alpha=\frac{D h}{v_{0}}$. Because the focus of this problem is the concentration of salt which is very close to the semipermeable membrane, it is necessary to consider the semi-infinite region where the concentration away from the membrane is the concentration of the solution before flowing into the filter, that is, $c_{0}$. Therefore, we have

$$
C(0, y)=c_{0}, C(x, \infty)=c_{0}, y=0
$$

At the boundary $y=0$ there is no salt flow. The boundary $y=0$ is the position of the semipermeabel membrane. However, because at $y=0$ there is vertikal velocity, we have diffusion flux $J_{\text {diff }}$ of salt and advection flux $J_{a d v}$ of salt:

$$
J=J_{\text {diff }}+\mathrm{J}_{\mathrm{adv}} \text {. }
$$

Here

$$
J_{\text {diff }}=-D \frac{\partial C}{\partial y},
$$

and

$$
J_{a d v}=-q C(x, 0),
$$

where $q$ is the water flow rate through the membrane. At the membrane $y=0$, we have $J(x, 0)=0$ so we obtain:

$$
-D \frac{\partial C}{\partial y}(x, 0)-q C(x, 0)=0 .
$$

We can also model the excess concentration as follows:

$$
c(x, y)=C(x, y)-c_{0} .
$$

Substitution of equation (10) into equation (4) leads to

where

$$
y \frac{\partial c}{\partial x}=\alpha \frac{\partial^{2} c}{\partial y^{2}}, \alpha=\frac{D h}{v_{0}}
$$




$$
c(0, y)=0, c(x, \infty)=0, y=0 .
$$

From equation (9) we obtain

$$
-D \frac{\partial c}{\partial y}(x, 0)=q C(x, 0)
$$

an based on equation (10) we have

$$
-D \frac{\partial c}{\partial y}(x, 0)=q\left(c(x, 0)+c_{0}\right) .
$$

Assuming that $c(x, 0)<<c_{0}$ we can approximate the boundary condition (12) as

$$
-D \frac{\partial c}{\partial y}(x, 0)=q c_{0} \text {. }
$$

Using the stretching transformations following Fulford and Broadbridge [10], we obtain the concentration of salt at the $x$ direction on the semipermeable membrane is

$$
C(x, 0)=1.53611715\left(\frac{q c_{0}}{D}\right)\left(\frac{D h}{v_{0}}\right)^{\frac{1}{3}} x^{\frac{1}{3}}+c_{0} .
$$

Equation (15) is the solution to our reverse osmosis problem. Note that there is a typographical error in the solution of Fulford and Broadbridge [10]: Fulford and Broadbridge calculated the coefficient to be 0.64 , but in fact it is 1.53611715 .

\section{Results}

Suppose that we have $q=10^{-3}, v_{0}=10^{-3}, D=10^{-9}$, and $h=10^{-3} \mathrm{~cm}$. Equation (15) gives

$$
\frac{C(x, 0)}{c_{0}}=1.53611715\left(10^{3}\right) x^{\frac{1}{3}}+1 .
$$

Based on equation (16), concentration increases with distance along the place, but this increase occurs slowly due to the factor $x^{\frac{1}{3}}$. We observe in Figure 3 with the parameter values selected, the increase in salt solution concentration is proportional $(300$ in $200 \mathrm{~cm})$. This concentration increases if the flow rate $q$ is increased through a semipermeable membrane. 


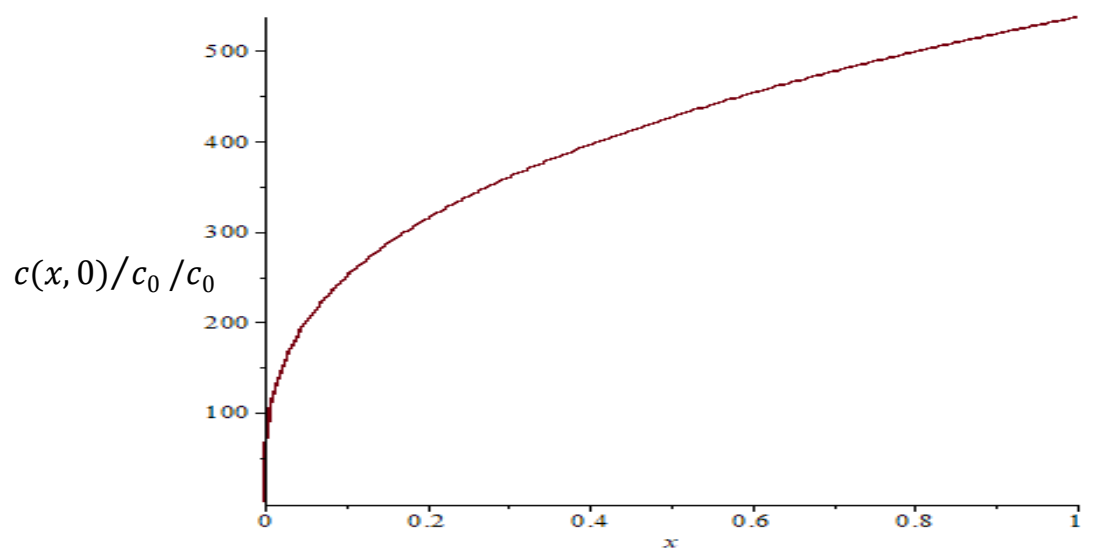

Fig. 3. Concentration $\mathrm{C} / \mathrm{c}_{0}$ at position $x$ and $y=0$ for $q=10^{-3}, v_{0}=10^{-3}, D=10^{-9}$, and

$$
h=10^{-3} \mathrm{~cm} \text {. }
$$

\section{Conclusion}

We have investigated that analytical methods can predict salt solution concentrations in reverse osmosis systems in water purification. Before using analytical methods, the writer needed to convert partial differential equations into ordinary partial differential equations by using analytical stretching transformation methods. This analytical method successfully solves the reverse osmosis problem.

Acknowledgments. The authors thank Universitas Sanata Dharma for the financial support.

\section{References}

[1] G. S. Garud, R. M., Kore, S. V., Kore V. S., Kulkarni, “A short review on process and applications of reverse osmosis,” Univers. J. Environ. Res. Technol., vol. 1, pp. 233-238, 2011.

[2] A. M. Abdallah, H., Shalaby, M. S., Saad, M. A., Shaban, "Supporting polyvinylchloride polymeric blend membrane with coated woven fabric," J. Membr. Sci. Res., vol. 4, pp. 174-180, 2018. [3] R. F. Spellman, Reverse Osmosis: A Guide for the Nonengineering Professional. Boca Raton, London, New York: Taylor and Francis, 2016.

[4] S. J. Wimalawansa, "Purification of contaminated water with reverse osmosis: effective solution of providing clean water for human needs in developing countries," Int. J. Emerg. Technol. Adv. Eng., vol. 3, pp. 75-89, 2013.

[5] I. G. Wenten, Teknologi membran: prospek dan tantangannya di indonesia (in Indonesian language). Bandung: Forum Guru Besar Institut Teknologi Bandung, 2016.

[6] N. Kumar, A., Jaiswal, D. K., Kumar, "Analytical solutions to one-dimensional advectiondiffusion equation with variable coefficients in semi-infinite media.," J. Hydrol., vol. 380, pp. 330-337, 2010.

[7] A. Djordjevich, A., Savović, S., Janićijević, "Explicit finite-difference solution of two-dimensional solute transport with periodic flow in homogenous porous media," J. Hydrol. Hydromechanics, vol. 65, pp. 426-432, 2017. 
[8] A. Mahmoodi, K., Ghassemi, H., Heydarian, "Solving the nonlinear two-dimension wave equation using dual reciprocity boundary element method," Int. J. Partial Differ. Equations Appl., vol. 5, pp. 19$25,2017$.

[9] M. T. Tirabassi, T., Buske, D., Moreira, D. M., Vilhena, "A two-dimensional solution of the advection-diffusion equation with dry deposition to the ground.," J. Appl. Meteorol. Climatol., vol. 47, pp. 2096-2104, 2008.

[10] P. Fulford, G. R., Broadbridge, Industrial Mathematic: Case Studies in the Diffusion of Heat and Matter. Cambridge: Cambridge University Press, 2002. 